\title{
Tea (Camellia sinensis O. Kuntze) Seed Oil and Health Properties
}

\author{
Fatih Seyis (Corresponding author) \\ Field Crops Department, Faculty of Agriculture and Natural Sciences, \\ Recep Tayyip Erdogan University, PO box 53300, Rize, Turkey \\ E-mail: fatih.seyis@erdogan.edu.tr \\ Emine Yurteri \\ Field Crops Department, Faculty of Agriculture and Natural Sciences, \\ Recep Tayyip Erdoğan University, PO box 53300, Rize, Turkey \\ E-mail: emine.yurteri@erdogan.edu.tr \\ Aysel Ozcan \\ Field Crops Department, Faculty of Agriculture and Natural Sciences, \\ Recep Tayyip Erdoğan University, PO box 53300, Rize, Turkey \\ E-mail: aysel.ozcan@erdogan.edu.tr
}

\begin{abstract}
Tea Oil has a mild fragrant flavor that goes with anything. It's not a heavy oil like Olive Oil, but thinner - more like almond oil. If the taste or "oiliness" of olive oil overpowers your food. Along with its mild taste and pleasant tea-like aroma, this oil touts impressive health benefits. Tea seed oil has a high smoke point, contains more monounsaturated fatty acids than olive oil, contains fewer saturated fatty acids than olive oil, contains high levels of Vitamin E, polyphenol antioxidants and both Omegas 3 and 6, but has less Omega 6 and Polyunsaturated Fats than olive oil. Health Benefits of tea seed oil are: it can be applied topically and consumed internally to obtain its health benefits, camellia oil can be used for skin, hair, has anti-cancer effects, effects boost immunity and reduces oxidative stress. Camellia oil is used for a variety of other purposes, for example for cooking, as machinery lubricant, as ingredient in beauty products like night creams, salves, in hair care products and perfumes and is used to coat iron products to prevent rusting.
\end{abstract}

Keywords: tea, seed oil, health

DOI: $10.7176 / \mathrm{JSTR} / 5-3-11$

\section{Introduction}

Like other genera of Camellia (from Theaceae family), the tea plant (C. sinensis) produces large oily seeds. In some countries where tea seed oil is abundantly available, it has been accepted as edible oil (Sahari et al., 2004). Tea seed oil is an edible oil cold-pressed from the seeds of Camellia oleifera and Camellia sinensis. In Chinese herbal medicine, tea seed oil is considered a superior nutritional dietary supplement that benefits the digestive system, reduces bad cholesterol, lowers blood pressure, regulates the nervous system, and strengthens the immune system. Tea seed oil is recommended by the Food and Agriculture Organization of the United Nations as a highquality, healthy vegetable oil because of its nutritional value, which is comparable to olive oil in terms of its high oleic acid content, low saturated fat, high antioxidants, and excellent storage qualities.

There are several reports that the oil content of tea seed is about 30-32\% (when computing the kernel and seed ratio, the value was 20\%), remaining a liquid even at refrigeration temperature, and has a high organoleptic acceptability (Ravichandran, 1993; Sahari et al., 2004). The predominant fatty acid in tea seed oil, as determined by GC/MS, is monounsaturated fatty acid, e.g. oleic acid, followed by the poly unsaturated fatty acid (PUFA), linoleic acid. Tea seed oil is high quality cooking oil, like olive oil, and it can be stored well at room temperature. Tea seed oil is reputed to lower blood pressure and cholesterol level, to have a high content of antioxidants, and to be a rich source of emollients for skin care and to minimize signs of aging (Fattahi-Far et al., 2006). The health properties of tea seed oil will be discussed in detail. 


\section{Tea Seed}

Tea (Camellia sinensis (L.) O. Kuntz) is one of the most economically essential beverage crops in all over the world and is considered to be the national drink. However, due to the uprooting of old seed plantations in different parts of the country; conservation of valuable tea germplasm in the form of seeds has assumed considerable importance (Katsuo et al., 1970ab; Chen et al., 2012). The seed of tea plant consists of an embryo surrounded by several extra embryonic layers as the covering and is usually develops from the fusion of the nuclei of male and female gametes. Tea seeds are borne in capsules, each containing one to three seeds. Seed is a means for reproduction and always possess variation within groups of seedlings. In nature, variability has an essential role in the production of the crop with quality and quantity. The broad genetic elasticity within the seed population provides the genetic material that allows the continued adaptation of a particular species to the environment.

\subsection{Tea Seed Oil}

All species of the Camellia genus produce an oleagenous seed. Crude edible oil was produced from tea seed in native mills in West Bengal, Himachal Pradesh and Assam and in the Northern region of Indochina (Owuor et al., 1985). Tea seed oil has been produced on commercial scale in China where in 1958, 180,000 tons of the oil) was produced (Sengupta et al., 1986).

The oil extracted from the seeds of Camellia species both cultivated as well as other species is termed as tea seed oil. Though C. sinensis is cultivated mostly for producing tea of commerce, oil is not usually obtained from this species. Commercial production of oil is derived from species like C. sasanqua, C. japonica, C. tenuifolia and C. oleifera. Seeds of different Camellia species contain $20-70 \%$ oil which is comparable to olive (Olea europaea) oil in its quality (Table 1 and 2). It could therefore be a potential substitute for olive oil as well as other edible oils. The tea seed oil is yellow coloured, free flowing, has pleasant odour and can be stored for 3 months at room temperature without loss in quality (Roberts and De Silva, 1972). Fatty acid composition of C. sinensis seed oil consisted of $21.5 \%$ palmitic acid, 2.9\% stearic acid (Rajaei et al., 2008), 56\% oleic acid, 22\% linoleic acid and $0.3 \%$ linolenic acid (Sahari et al., 2004). The major fatty acid (50\% of the total oil) in the C. sinensis seed oil was oleic acid (Rajaei et al., 2005). Therefore, with regard to oleic acid, C. sinensis seed oil can be ranked between sunflower and olive oil (Sahari et al., 2004).

The proportions of UFAs and SFAs in the extracted oils were 58.1-71.7\% and 17.4-23.7\%, respectively (Rajaei et al., 2005). This oil had little tendency to dry because of the low C18:2 and C18:3 contents, which cause polymerization (Sahari et al., 2004).

Table 1. Oil content in Camellia species and other oil crops

\begin{tabular}{|l|c|}
\hline Species & Per cent oil (Dry weight basis) \\
\hline C. sasanqua & $60-70$ \\
\hline C. japonica & $66-70$ \\
\hline C. oleifera & $40-50$ \\
\hline C. tenuifolia & $40-50$ \\
\hline C. sinensis (Common tea) & $20-30$ \\
\hline Groundnut & $44-50$ \\
\hline Olive (Common olive) & $15-40$ \\
\hline Sunflower & $35-48$ \\
\hline Mustard & $30-48$ \\
\hline
\end{tabular}

Owuor et al. (1985); Kamenga (1985); Anonymous (1950)

In previous studies, in $C$. oleifera Abel oil, the average content of MUFA, PUFA and SFA were 51.06\%, 27.86\% and $20.67 \%$ and the major component were oleic, linoleic, palmitic and stearic acids, respectively (Hua et al., 2008). Also, UFAs content is $75.89 \%$, in which the content of predominate oleic and linoleic acid is $73.83 \%$ (Weiming et al., 1992). 
Table 2. Fatty acids profiles of C. sinensis seed oil

\begin{tabular}{|l|l|}
\hline Fatty acids & Tea seed oil (\%) \\
\hline C16:0 & 16.50 \\
\hline C18:0 & 3.33 \\
\hline C18:1 & 65.97 \\
\hline C18:2 & 22.17 \\
\hline C18:3 & 0.30 \\
\hline C20:0 & 0.53 \\
\hline PUFA & 22.47 \\
\hline
\end{tabular}

\subsection{Health benefits of tea seed oil}

Weiming et al. (1992)

The beverage tea plant is mainly cultivated for its prolific vegetative growth. Tea plant produced large amounts of oil (30-32\%) moreover; its seeds act as functional product with various applications. This oil is one of the important vegetable oils because of the high unsaturated fatty acids, especially essential linoleic acid and low content of saturated fat (Sahari and Amooi, 2013). The oil of tea seed also lower blood pressure and cholesterol level, and has functional effects against several degenerative pathologies, including cardiovascular diseases and cancers. Tea seed oil is a high quality cooking oil, like olive oil; it has an excellent storage quality due to a high content of polyphenols as antioxidant agents. Tea seed oil also is a good raw material for producing cocoa butter equivalent and margarine.

Tea seed oil was also traditionally applied as a medicine for stomach ache and burning injuries in China. Lee et al. (2007) indicated that saponin in tea seed could lower the serum levels of cholesterol, triglycerides, and low densitylipoprotein in rats (Lee and Yen, 2006; Lee et al., 2007). Tea seed oil is reputed to lower blood pressure and cholesterol level, to have a high content of antioxidants (polyphenols, carotenoids and vitamin E), and to be a rich source of emollients for skin care, and to minimize signs of aging (Fazel et al., 2008; Fattahi-far et al., 2006). The formation of intercellular reactive oxygen species (ROS), inhibit low density lipoprotein (LDL) oxidation, and protect lymphocytes against $\mathrm{H} 2 \mathrm{O} 2$-induced genetic injury could be reduced by the methanol extracts of tea seed oil (METSO) (Lee et al., 2007). Tea oil is a good raw material for industrial use and is used in manufacturing of soap, margarine, hair oil, lubricants, and paint, and also in the synthesis of other high-molecular weight compounds and rustproof oil (Rajaei et al., 2005).

\section{Conclusion}

All species of the genus Camellia produce oil-rich seeds as by-product. From nutrition point of view, tea seed oil is one of the important vegetable oils because of the high contents of unsaturated fatty acids, especially essential linoleic acid, which reported to lower blood cholesterol level. In general, chemical properties, fatty acid composition and triacylglycerol species of this oil determine their best nutritional and commercial uses. It also has a little amount of linolenic acid, which is an important factor in rancidity and off-flavor of oils during storage time (Sahari and Amooi, 2013). Tea seed oil is also high quality cooking oil, like olive oil, and has excellent storage quality to have a large amount of carotenoids, polyphenols and vitamin E, as natural antioxidants. Tea seed oil possesses antioxidant properties which was almost equal with sesame seed oil, and could be used an as alternative natural antioxidant. Therefore, it could be used directly as a natural antioxidant and functional product for increasing food stability. Furthermore, tea seed oil can be improved the application of oils in industrial conditions, for example margarine production, cocoa butter replacer production and formulations of chocolate and confectionary.

The tea plantations in Turkey are approximately 83 ha (ÇAYKUR, 2016). After improving the yield of present plantations and after determination of suitable genotypes some parts of these plantations could be separated for tea oil and tea honey production in Turkey.

\section{References}

Anonymous (1950). Camellia. In "Wealth of India" 2: 27-50.

Chen, H., Pritchard, H.W., Seal, C.E., Nadarajan, J., Li, W., Yang, S. \& and Kranner I. (2012). Post desiccation germination of mature seeds of tea (Camellia sinensis L.) can be enhanced by pro-oxidant treatment, but partial desiccation tolerance does not ensure survival at $-20^{\circ} \mathrm{C}$. Plant Science, 184:36-44.

\section{ÇAYKUR. 2016. İstatistik Bülten. ÇAYKUR. (Statistical Bulletin, ÇAYKUR)}

Fattahi-far, E., Sahari, M.A. \& Barzegar, M. (2006). Interesterification of tea seed oil and its application in margarine production. Journal of the American Oil Chemists' Society, 83: 841-845.

$86 \mid \mathrm{P}$ a g e

www.iiste.org 
Fazel, M., Sahari, M.A. \& Barzegar, M. (2008). Determination of main tea seed oil antioxidants and their effects on common kilka oil. International Food Research Journal, 15: 209-217.

Hua, G., Jianping, Z., Junwu, L., Huiyuan, T. \& Dongmin L. (2008). Fatty acid composition analysis of tea seeds oil. China Oils and Fats.

Kamanga, K.D.E. (1985). Tea seed oil project. TRFCA. Quarterly Newsletter, 79: 29-30.

Katsuo, K, Toyao, T. \& Kayumi, S. (1970a). The germination tea seed (I): Relation of picking period and conditions for storage to the seed germination. Study of Tea, 39:14-19.

Katsuo, K. Toyao, T. \& Kayumi S. (170b). The germination of tea seed (II): Accelerating effectof pretreatment on the tea seed germination. Study of Tea, 39:20-25.

partial desiccation tolerance does not ensure survival at $-20^{\circ} \mathrm{C}$. Plant Science. 2012;184:36-44.

partial desiccation tolerance does not ensure survival at $-20^{\circ} \mathrm{C}$. Plant Science.

2012;184:36-44.

Lee, C.P., Shih, P.H., Hsu, C.L. \& Yen, G.C. (2007). Hepatoprotection of tea seed oil (Camellia oleifera Abel.) against CCl4-induced oxidative damage in rats. Food Chem Toxicol, 45: 888-895.

Lee, C.P. \& Yen, G.C. (2006). Antioxidant activity and bioactive compounds of tea seed (Camellia oleifera Abel.) oil. Journal of Agriculture and Food Chemistry, 54: 779-784.

Owuor, P.O., Chauanji, A.M. \& Manavu, R.M. (1985). Chemical studies of the Kenian tea seeds I. Physical and chemical characteristics of the Kernel oil. Tea, 6 (1):23-28.

Rajaei, A., Barzegar, M. \& Yamini, Y. (2005). Supercritical fluid extraction of tea seed oil and its comparison with solvent extraction. European Food Research and Technology, 220: 401-405.

Rajaei, A., Barzegar, M. \& Sahari, M.A. (2008). Comparison of antioxidative effect of tea and sesame seed oils extracted by different methods. J Agric Sci Technol, 10: 345-350.

Ravichandran, R. (1993). Fat stability and amino acids in south Indian tea seeds. International Journal of Food Science and Technology, 28: 639-646.

Roberts, G.R. \& De Silva, U.L.L. (1972). Products from tea seed 1. Extraction and roperties of oil. Tea, Q. 43 (3): $88-90$.

Sahari, M.A., Atai, D. \& Hamedi, M. (2004). Characteristics of tea seed oil in comparison with sunflower and olive oils and its effect as a natural antioxidant. Journal of the American Oil Chemists' Society, 81: 585588 .

Sahari, M.A. \& Amooi, M. (2013). Tea seed oil: Extraction, compositions, applications, functional and antioxidant properties. Academia Journal of Medicinal Plants, 1(4): 068-079.

Sengupta, C., Sengupta, A. \& Ghosh, A. (1976). Triglyceride composition of tea seed oil. J Sci Fd Agric, 27: 1115-1122.

Weiming, Z., Guorong, W., Botao, Z., Hong, W. \& Shirong, C. (1992). Studies on the chemical components of tea seed oil and cake. Nanjing Normal Univ Nat Sci, Edition 3. 